\title{
Pre-eclamptic Fetal Programming Alters Neuroinflammatory and Cardiovascular Consequences of Endotoxemia in Sex-Specific Manners
}

\author{
Salwa A. Abuiessa, (1) Abdalla M. Wedn, Sahar M. El-Gowilly, Mai M. Helmy, \\ and (1) Mahmoud M. El-Mas \\ Department of Pharmacology and Toxicology, Faculty of Pharmacy, Alexandria University, Alexandria, Egypt
}

Received November 25, 2019; accepted February 12, 2020

\begin{abstract}
Pre-eclampsia (PE)-induced fetal programming predisposes offspring to health hazards in adult life. Here, we tested the hypothesis that pre-eclamptic fetal programming elicits sexually dimorphic inflammatory and cardiovascular complications to endotoxemia in adult rat offspring. PE was induced by oral administration of L-NAME (50 mg/kg per day for seven consecutive days) starting from day 14 of conception. Cardiovascular studies were performed in conscious adult male and female offspring preinstrumented with femoral indwelling catheters. Compared with non-PE male counterparts, intravenous administration of lipopolysaccharide (LPS, $5 \mathrm{mg} / \mathrm{kg}$ ) to PE male offspring caused significantly greater 1) falls in blood pressure, 2) increases in heart rate, 3) rises in arterial $\mathrm{dP} / \mathrm{dtmax}$, a correlate of left ventricular contractility, and 4) decreases in time- and frequency-domain indices of heart rate variability (HRV). By contrast, the hypotensive and tachycardic actions of LPS in female offspring were independent of the pre-eclamptic state and no clear changes in HRV or $\mathrm{dP} / \mathrm{dtmax}$ were noted. Measurement of arterial baroreflex activity by vasoactive method revealed no sex specificity in baroreflex dysfunction induced by LPS. Immunohistochemical studies showed increased protein
\end{abstract}

expression of toll-like receptor 4 in heart as well as in brainstem neuronal pools of the nucleus of solitary tract and rostral ventrolateral medulla in endotoxic PE male, but not female, offspring. Enhanced myocardial, but not neuronal, expression of monocyte chemoattractant protein-1 was also demonstrated in LPS-treated male offspring. Together, pre-eclamptic fetal programming aggravates endotoxic manifestations of hypotension and autonomic dysfunction in male offspring via exacerbating myocardial and neuromedullary inflammatory pathways.

\section{SIGNIFICANCE STATEMENT}

Current molecular and neuroanatomical evidence highlights a key role for pre-eclamptic fetal programming in offspring predisposition to health hazards induced by endotoxemia in adult life. Pre-eclampsia accentuates endotoxic manifestations of hypotension, tachycardia, and cardiac autonomic dysfunction in male offspring via exacerbating myocardial and central inflammatory pathways. The absence of such detrimental effects in female littermates suggests sexual dimorphism in the interaction of pre-eclamptic fetal programming with endotoxemia.

\section{Introduction}

Pre-eclampsia $(\mathrm{PE})$ is a new-onset gestational hypertensive status that is commonly associated with aggravated proteinuria, renal insufficiency, and impaired liver function (Brown et al., 2018). PE complicates almost $5 \%$ of all pregnancies (Morton, 2016) and negatively affects maternal and fetal health (Fox et al., 2019). Adverse fetal consequences involve antenatal risks of intrauterine growth restriction, preterm birth, and possibly fetal death in utero (Haddad et al., 2004; Madazli et al., 2014; Rezk et al., 2015). Moreover, in utero exposure to hypertensive state during pregnancy can

Supported by the Science and Technology Development Fund, Egypt (STDF Grants No. 14895 and 37026).

The authors declare no conflict of interest.

https://doi.org/10.1124/jpet.119.264192. result in long-term cardiovascular sequelae in offspring, including early onset hypertension, and increased risk of ischemic heart disease and stroke (Davis et al., 2012). The term "fetal programming" decribes developmental fetal adaptations during pregnany with consequent cardiovascular, metabolic, and endocrine disorders in adulthood (Godfrey and Barker, 2001). During PE, the harsh intrauterine environment, induced by angiogenic, inflammatory, and hypoxic insults, causes genomic alterations in mother and fetus that would ultimately modify the expressed phenotype (Stojanovska et al., 2016). Fetal programming in response to prenatal stress alters offspring cardiovascular health. Litters of prenatally stressed rats elicit exaggerated and more sustained elevations in $\mathrm{BP}$ and its variability, with female offspring showing more dramatic effects compared with their male counterparts (Igosheva et al., 2004).

ABBREVIATIONS: BP, blood pressure; BRS, baroreflex sensitivity; FFT, fast-Fourier transform; HF, high frequency; HR, heart rate; HRV, heart rate variability; LF, low frequency; LPS, lipopolysaccharide; LV, left ventricular; MAP, mean arterial pressure; MCP-1, monocyte chemoattractant protein-1; NTS, nucleus tractus solitarious; PE, pre-eclampsia; rMSSD, oot mean square of successive beat-to-beat differences in R-R interval durations; RVLM, rostral ventrolateral medulla; SBP, systolic blood pressure; SDNN, SD of beat-to-beat intervals; TLR-4, toll-like receptor 4. 
Various animal models of PE are available. An ultra-lowdose of LPS has been used to replicate PE manifestations in rats including hypertension, platelet coagulopathy, and glomerular fibrinogen deposition (Faas et al., 1994). Alternatively, chronic inhibition of nitric oxide synthase with L-NAME ( $\mathrm{N} \omega$-Nitro-L-arginine methyl ester hydrochloride) in pregnant rats results in hypertension accompanied by thrombocytopenia, reduced glomerular filtration rate, proteinuria, and intrauterine growth restriction (McCarthy et al., 2011; Marshall et al., 2018). Unlike LPS model of PE, serumfree fatty acid, hepatic, and placental fatty deposition are elevated in L-NAME treated dams (Han et al., 2015). This could be related to different pathogenic pathways in the two models, with primarily fatty acid oxidative disorders in L-NAME model and aberrant inflammatory cascade through the activation of the NF- $\kappa$ B signaling pathway, and damage of endothelial cells in LPS model (Ding et al., 2015). Physiologic and histologic perturbations induced by gestational L-NAME largely resemble clinical manifestations of PE. This includes 1) elevated placental expression of inflammatory, antiangiogenic, and apoptotic factors; 2) reduced antioxidant activity; and 3) increased glomerular area, capillary structure disorder, abnormal protein cast, and reduced nephron numbers (Amaral et al., 2018; Shu et al., 2018; Chen et al., 2019; Zheng et al., 2019).

Sepsis is a fatal organ dysfunction caused by disrupted host response to infection. Endotoxemia is employed to model the hyperinflammatory state characteristic of early sepsis (Dickson and Lehmann, 2019). LPS from Gram negative pathogens is classically used to fire the immune responses, leading to hyperinflammation and microcirculatory perturbations (Dickson and Lehmann, 2019). Experimental data from our laboratory (Sallam et al., 2016) and others (Zila et al., 2015) demonstrated that endotoxemia elicits hypotensive, tachycardic, and cardiac autonomic depressant consequences. These harmful derangements could be related to the excessive generation of inflammatory cytokines, which induce mitochondrial damage, cripple calcium homeostasis, and perturb the neuroautonomic tone (Flierl et al., 2008). We and others have previously reported sexual dimorphism in immune response to endotoxemia, with females experiencing less adverse clinical outcomes than age-matched males (Saia et al., 2015; Klein and Flanagan, 2016; El-Lakany et al., 2018).

Although sex differences in cardiovascular consequences of endotoxemia have been demonstrated, the influence of $\mathrm{PE}$ on cardiovascular sequale of endotoxemia in adult offspring remains largely obscure. This prompted us to test the novel hypothesis that PE fetal programming elicits sexually dimorphic inflammatory, cardiovascular, and autonomic complications in response to endotoxemia in adult rat offspring. Endotoxemia was induced by intravenous administration of a single dose of LPS ( $5 \mathrm{mg} / \mathrm{kg}$ ) (Lv et al., 2006) in conscious agematched PE and non-PE male and female offspring. Cardiac autonomic activity was assessed by: 1) HRV analysis, which determines global autonomic control and cardiac sympathovagal balance (Stein et al., 1994; El-Mas and Abdel-Rahman, 2013), and 2) arterial baroreceptor testing, which symbolizes reflex control of cardiac autonomic function (Smyth et al., 1969). Molecular studies were undertaken to assess inflammatory state in myocardial tissues as well as in brainstem neuronal pools of the nucleus tractus solitarious (NTS) and rostral ventrolateral medulla (RVLM). The latter are brainstem nuclei that play critical roles in cardiovascular and cardiac autonomic control (Saha, 2005) as well as in central processing of central neuroinflammatory signals including those triggered by the endotoxic insult (Sirivelu et al., 2012; Sallam et al., 2019).

\section{Materials and Methods}

Adult Wistar rats (170-240 g; Faculty of Pharmacy Animal Facility, Alexandria University, Alexandria, Egypt) were used in this study. All experiments were approved by the Institutional Animal Care and Use Committee and carried out in accordance with the Declaration of Helsinki and the Guide for the Care and Use of Laboratory Animals (Institute of Laboratory Animal Resources, 1996) as adopted and promulgated by the National Institutes of Health. This protocol was approved by the Institutional Animal Care and Use Committee, Alexandria University, Egypt (Approval No. AU0620191113262).

Induction of Pre-eclampsia. Pregnancy was induced by introducing adult nulliparous female rats to larger males (ratio 1:1) to allow for overnight mating. The day of conception was determined by checking the spermatozoa in the vaginal lavage or detection of a vaginal plug. $\mathrm{PE}$ was induced by daily oral administration of $50 \mathrm{mg} / \mathrm{kg}$ of L-NAME for 7 days starting from day 14 of conception (Pandhi et al., 2001). The development of PE was assessed by measurement of systolic blood pressure (SBP) by the tail cuff technique as well as by assessment of urinary protein level. Ten weeks after spontaneous delivery, adult male and female offspring were processed for measurement of SBP and proteinuria. Rats were then subjected to intravascular cannulation for undertaking cardiovascular studies as detailed below.

Tail-Cuff Plethysmography. Tail-cuff measurements of SBP were conducted in conscious pregnant rats and adult offspring using a computerized data acquisition system with LabChart-7 pro software (Power Laboratory 4/30, model ML866/P; AD Instruments, Bella Vista, Australia) as described in our previous studies (El-Mas et al., 2015). Heart rate (HR) was computed from BP waveforms and displayed on another trace of the recording system. A specialized tail cuff and pulse transducer (Pan Laboratory, Spain) were used for SBP measurement depending on the periodic obstruction of tail arterial blood flow. SBP was measured three or four times and values were averaged to the mean.

Urine Collection and Protein Analysis. For 24-hour urine collection, pregnant dams (gestational day 20) and adult offspring (10 weeks old) were housed in metabolic cages with stainless steel mesh wire bottom. Rats were allowed ad libitum access to standard rat chow and water. Urine samples were collected under light mineral oil and kept at $-80^{\circ} \mathrm{C}$ until processed (El-Mas and Abdel-Rahman, 2007). Urinary protein levels were assessed using pyrogallol red method (Zhang et al., 2009) using Cromatest standard kit (LiNEAR Chemicals, Spain) according to the manufacturer's guidelines.

Intravascular Cannulation. Rats were anesthetized with thiopental $(50 \mathrm{mg} / \mathrm{kg}$, i.p.) and femoral artery and vein cannulation was performed as described previously (el-Mas et al., 1997; El-Mas and Abdel-Rahman, 1999; El-Mas et al., 2006, 2009). As detailed below, full cardiovascular and autonomic investigations were conducted 2 days later in conscious, freely moving rats.

Time-Domain Analysis of HRV. Two time-domain measures of the cardiac autonomic activity were used, the SD of beat-to-beat intervals (SDNN) and the root mean square of successive beat-to-beat differences in R-R interval durations (rMSSD) (Stein et al., 1994; Omar and El-Mas, 2004). The R-R intervals were computed from the $\mathrm{HR}$ (i.e., the reciprocal of the HR in milliseconds). The SDNN is comparable to the total power of the spectrum of R-R variability, which measures the overall autonomic balance of the heart. The rMSSD is largely validated as a measure of the parasympathetic input to the heart and, therefore, correlates with the high-frequency power of the spectrum (Stein et al., 1994; Sgoifo et al., 1997). SDNN and rMSSD 
were measured before (baseline) and at 15-minute intervals after drug treatments. For each time point, the 5 -minute values of each variable were averaged.

Frequency-Domain Analysis of HRV. Spectral hemodynamic fluctuations, quantitative indices of cardiac autonomic control (Stein et al., 1994; El-Mas and Abdel-Rahman, 2007; Sallam et al., 2016), were used to reflect changes in sympathetic and vagal outflows. Hemodynamic variability was analyzed in the frequency domain using fast-Fourier transform (FFT) algorithms of R-R data series. The FFT algorithm for direct transformation of data points into power spectral density graphs was used. Data were interpolated to obtain equally spaced samples with an effective sampling frequency of $10 \mathrm{~Hz}(0.1$ seconds). A second-order interpolation was used to fit a smooth curve to the existing data points and to produce smoother visual representation of data. The evenly spaced (equidistant) sampling allowed direct spectral analysis using the FFT algorithm. Spectra were integrated into two specific frequency bands, low-frequency (LF) $(0.25-0.75 \mathrm{~Hz})$ and high-frequency $(\mathrm{HF})(0.75-3 \mathrm{~Hz})$ bands and expressed in normalized units $\left(\mathrm{LF}_{\mathrm{nu}}\right.$ and $\left.\mathrm{HF}_{\mathrm{nu}}\right)$. Spectral data were estimated before (baseline) and at 15-minute intervals after drug treatments. For each time point, the 5 -minute values of each variable were averaged.

Baroreflex Testing. Baroreflex sensitivity (BRS) was evaluated using the vasoactive method (Smyth et al., 1969; El-Mas et al., 2012). This method is based on the measurement of bradycardic and tachycardic responses to peripherally induced increases and decreases in BP evoked by bolus intravenous injections of randomized doses of phenylephrine $\left(\mathrm{BRS}_{\text {phenylephrine }}\right)$ and sodium nitroprusside $\left(\mathrm{BRS}_{\text {nitroprusside }}\right)$ (1-16 $\mu \mathrm{g} / \mathrm{kg}$ each), respectively, at 5-minute intervals. The relationship between changes in MAP and associated reciprocal changes in HR were assessed by regression analysis for individual animals. The slope of the dynamic part of the curve expressed as beats per minute per millimeters of $\mathrm{Hg}$ was considered as an index of BRS.

Immunohistochemistry. The technique described in our previous studies (El-Mas et al., 2006; Helmy et al., 2015; Sallam et al., 2019) was employed for immunohistochemical measurement of protein expression of TLR- 4 and MCP-1 in rat heart (anterior portion of the apex) and brainstem pools of NTS and RVLM. Cardiac and brain tissues were fixed in $10 \%$ formaldehyde solution and embedded in paraffin blocks. Sections $(5 \mu \mathrm{m})$ of heart or brainstem $(-12.0$ to $-12.48 \mathrm{~mm}$ relative to bregma, see Fig. 1) were cut and put on positively charged adhesive glass slides (Thermo Scientific, Berlin, Germany), then deparaffinized in xylene and rehydrated in a series of declining ethanol concentration (100\%, 95\%, and 70\%). Heat-induced epitope retrieval was performed by immersing slides in coplin jars containing $10 \mathrm{mM}$ citrate buffer solution and incubated in a microwave at power 100 for 1 minute then power 30 for 9 minutes. Endogenous peroxidases were blocked by $3 \%$ hydrogen peroxide for 10 minutes. The primary polyclonal antibodies, rabbit anti-TLR-4 (PA5-23124), rabbit anti-MCP-1 (PA5-34505) (ThermoFisher Scientific), were diluted (1:300) as instructed by the manufacturer, applied to the slides and then sections were incubated at $4^{\circ} \mathrm{C}$ overnight. The secondary antibody (horseradish peroxide conjugate) was applied for 30 minutes. The chromogen 3,3'-diaminobenzidine was prepared and applied as instructed by the manufacturer for protein visualization. Slides were counterstained with hematoxylin and dipped in ascending concentrations of alcohol and then xylene. Images were taken by OptikamB9 digital camera (Optika microscopes, Italy) and Fiji Image J software version 1.51n (National Institutes of Health, Bethesda, MD) was employed to quantitate the area fraction of chromogen 3,3'-diaminobenzidine-positive staining in cardiac and brainstem areas of NTS and RVLM.

Measurement of Serum MCP-1. Blood samples were withdrawn at the end of each experiment through the arterial catheter. The collected blood was centrifuged at $1118 \mathrm{~g}$ for 10 minutes and serum was stored at $-80^{\circ} \mathrm{C}$ for subsequent analyses. Serum MCP-1 was measured using the MCP-1 Rat Instant ELISA Kit (cat no: BMS631INS; ThermoFisher Scentific) according to the manufacturer's protocol.

Protocols and Experimental Groups. Eight groups of rats $(n=$ 8 each) preinstrumented with femoral indwelling catheters were used to test the effect of PE on cardiovascular, autonomic, and inflammatory manifestations of endotoxemia in male and female offspring. For each sex, four groups of rats were employed and categorized into: 1) saline-treated non-PE offspring, 2) LPS-treated non-PE offspring, 3) saline-treated PE offspring, and 4) LPS-treated PE offspring. On the experiment day, 2 days after femoral catheterization, the arterial

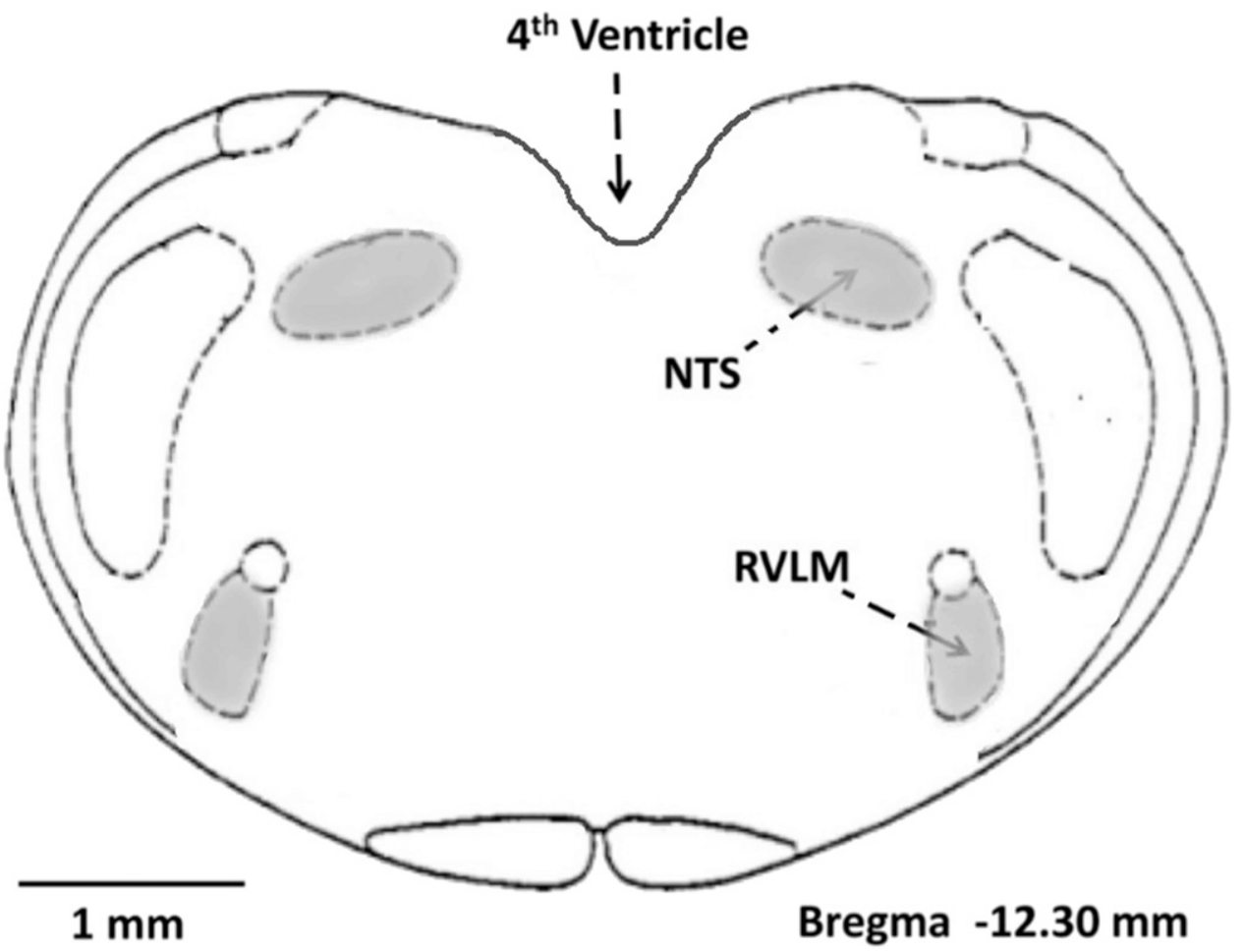

Fig. 1. Anatomic localizations of the NTS and RVLM of rat brainstem. 
catheter was connected to a BP transducer (model P23XL; Astro-Med, West Warwick, RI) that was connected through MLAC11 Grass adapter cable to a computerized data acquisition system with LabChart7 pro software (Power Laboratory 4/35, model ML866/P; AD Instruments Pty Ltd., Castle Hill, Australia). The HR was computed from BP waveforms and displayed on another trace of the recording system.

After an initial stabilization period of at least 30 minutes, rats were allocated to receive intravenous dose of LPS ( $5 \mathrm{mg} / \mathrm{kg}$ ) (Lv et al., 2006) or equal volume of saline over a period of 10 minutes. Hemodynamic monitoring continued for 2 hours post-treatment. Changes in MAP, $\mathrm{HR}$, time (SDNN, rMSSD) and spectral (total power and LF:HF ratio) measures of HRV, maximum rate of rise in left ventricular pressure (dP/dt max, a correlate of systolic contractility), were computed at 15minute intervals. The cumulative hypotensive effect of LPS over the entire 2-hour observation period of the study was computed by measuring the area under the curve of the hypotensive response for individual experiments using trapezoidal integration with zero line taken as the baseline (GraphPad Prism, version 3.02). This was computed by summing incremental areas of each trapezoid below the effect-time curve. After the 2-hour hemodynamic monitoring period, baroreflex curves were constructed by the vasoactive method as described above. Afterward, rats were euthanized using an overdose of thiopental $(100 \mathrm{mg} / \mathrm{kg})$, and hearts and brainstems were quickly removed, fixed in $10 \%$ formaldehyde solution, and embedded in a paraffin blocks within 24 hours to be used for immunohistochemical assessment of the protein expression of TLR-4 and MCP-1.

Drugs. Thiopental (Thiopental; Biochemie GmbH, Vienna, Austria), povidone-iodine solution (Betadine; Nile Pharmaceutical Co., Cairo, Egypt), Pencitard (NCPC North best Co., Hebei, China), Heparin (5000 IU/ml; Nile pharmaceutical Co.), LPS (from Escherichia coli 0111: B4), L-NAME, phenylephrine hydrochloride, and sodium nitroprusside (Sigma Aldrich, St. Louis, MO) were purchased from commercial vendors. All drugs were dissolved in saline.

Statistical Analysis. Values are expressed as means \pm S.E.M. The unpaired Student $t$ test or the one-way or repeated measures ANOVA followed by the Tukey's post hoc test was used to test for statistical significance. These analyses were performed by GraphPad InStat, software release 3.05. Probability levels less than 0.05 were considered significant.

\section{Results}

L-NAME Induces Pre-eclamptic Manifestations in Pregnant Female Rats. The daily treatment of pregnant rats with L-NAME ( $50 \mathrm{mg} / \mathrm{kg}$ per day) for seven consecutive days resulted in significant rises in $\mathrm{SBP}(137.2 \pm 5.2$ vs. 109.9 $\pm 1.7 \mathrm{~mm} \mathrm{Hg})$ and falls in HR $(336.1 \pm 7.7$ vs. $376.5 \pm 8.3$ beats/min) compared with saline-treated (non-PE) values. In 24-hour urine samples collected during the 20th day of gestation, significantly higher quantities of protein were detected in urine of PE (333.3 $\pm 51.1 \mathrm{mg} / \mathrm{dl})$ compared with non-PE females $(72.3 \pm 6.8 \mathrm{mg} / \mathrm{dl})$. This corresponds to approximately a fivefold increase in urine protein in PE females.

Effects of PE on Baseline Cardiovascular and Autonomic Functions in Male and Female Offspring. Table 1 summarizes baseline values of cardiovascular and autonomic functions in 10-week-old male and female offspring of $\mathrm{PE}$ and non-PE rats measured prior to intravenous administration of LPS or saline. The body weights of PE offspring (male or female) were significantly less than those of non-PE offspring of the same sex. Moreover, compared with their respective non-PE counterparts, male offspring of PE rats exhibited significantly 1) higher levels of proteinuria and SBP, and 2) lower baseline values of time (SDNN and rMSSD) and frequency (total power) indices of HRV. Unlike male offspring, none of these parameters was different between PE and non-PE female offspring (Table 1).

Pre-eclampsia Exacerbates Endotoxic Cardiovascular Manifestations in Male, but Not, Female Offspring. The effects of PE on cardiovascular and autonomic responses to endotoxemia in adult offspring are shown in Figs. 2-5. In all rat groups, intravenous infusion of LPS $(5 \mathrm{mg} / \mathrm{kg})$ resulted in significant falls in BP compared with values of saline-treated rats during the 2-hour duration of the experiment (Fig. 2, A and $\mathrm{C}$ ). The hypotensive action of LPS peaked at approximately 60 minutes and declined thereafter and was associated with synchronized rises in HR (Fig. 2, B and D). Both the hypotensive and tachycardic effects of LPS were significantly potentiated in PE male offspring compared with their non-PE counterparts. Such PE effects were not evident in female offspring, as similar decreases and increases in MAP and HR, respectively, were observed in $\mathrm{PE}$ and non-PE female offspring.

The areas under the LPS hypotensive curves, which represent cumulative falls in blood pressure over the 2-hour observation period, were computed to determine the intersex differences in the LPS response. Significantly greater areas under the curves of LPS hypotension were noted in female compared with male offspring of non-PE rats $(60 \pm 4$ vs. $48 \pm 3 \mathrm{~mm}$ Hg.min, $P<0.05$ ). In $\mathrm{PE}$ rats, by contrast, the accentuated LPS hypotension in male offspring resulted in significantly greater areas under the curves than those of female offspring (96 \pm 16 vs. $64 \pm 8 \mathrm{~mm} \mathrm{Hg.min,} P<0.05$ ).

Changes in time and frequency measures of HRV were determined as indices of cardiac autonomic activity. The data revealed that the time-domain parameter SDNN (Fig. 3A) as well as total power of the spectral profile (Fig. 4A), measures of the overall cardiac autonomic control, were significantly reduced at some time points after LPS injection in non-PE male offspring. These effects were more intensified in LPS-treated male offspring of PE mothers and remained so throughout the 2-hour observation period of the study (Figs. $3 \mathrm{~A}$ and 4A). Similar patterns of enhanced LPS-induced reductions in the time (rMSSD, Fig. 3B) and frequency domain $\left(\mathrm{HF}_{\mathrm{nu}}\right.$, Fig. 4B) indices of cardiac vagal activity in male offspring of $\mathrm{PE}$ compared with respective non-PE offspring. By contrast, spectral measures of cardiac sympathetic activity $\left(\mathrm{LF}_{\mathrm{nu}}\right.$, Fig. 4C) or sympathovagal balance (LF:HF ratio, Fig. 4D) were not altered by LPS in all male offspring preparations. In female offspring of $\mathrm{PE}$ or non-PE mothers, the time-domain parameters of SDNN (Fig. 3C) and rMSSD (Fig. 3D) showed slight, inconsistent, and similar reductions in response to LPS. None of the spectral indices of HRV was altered by LPS in female offspring (data not shown).

LPS did not affect the maximum rate of rise of $\mathrm{BP}$ waves $(\mathrm{dP} /$ dtmax, correlate of LV contractility) in non-PE male (Fig. 5A) or female (Fig. 5B) offspring. The changes caused by LPS in these parameters were not statistically different from those seen in saline-treated rats. However, a significant increase in $\mathrm{LV}$ contractility was observed in male offspring of $\mathrm{PE}$ endotoxic rats (Fig. 5A).

Modulation of Baroreflex Dysfunction in Endotoxic Rats by Pre-eclampsia is Not Sex Specific. Slopes of the baroreflex curves established by the vasoactive method are shown in Fig. 6. In male offspring, LPS caused significant

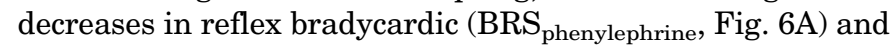

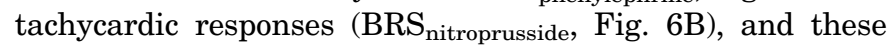
effects were independent of the pre-eclamptic state. In female 
TABLE 1

Effect of PE on body weight, proteinuria, and baseline (prior to LPS administration) cardiovascular parameters in adult offspring

Values are means \pm S.E.M.

\begin{tabular}{|c|c|c|c|c|}
\hline \multirow{2}{*}{ Parameter } & \multicolumn{2}{|c|}{ Males } & \multicolumn{2}{|c|}{ Females } \\
\hline & Non-PE & $\mathrm{PE}$ & Non-PE & $\mathrm{PE}$ \\
\hline Body weight (g) & $213.0 \pm 6.8$ & $194.1 \pm 4.5^{*}$ & $168.2 \pm 3.6$ & $144.3 \pm 3.9^{*}$ \\
\hline Proteinuria (mg/dl) & $221.5 \pm 13.4$ & $249.2 \pm 7.6^{*}$ & $94.2 \pm 9.3$ & $89.5 \pm 11.0$ \\
\hline $\mathrm{SBP}(\mathrm{mm} \mathrm{Hg})$ & $121.0 \pm 3.3$ & $133.3 \pm 3.9^{*}$ & $106.6 \pm 1.9$ & $112.3 \pm 2.1$ \\
\hline HR (beats/min) & $379.6 \pm 9.6$ & $382.9 \pm 11.8$ & $382.3 \pm 7.9$ & $402.2 \pm 11.0$ \\
\hline SDNN (ms) & $5.2 \pm 0.1$ & $3.7 \pm 0.3^{*}$ & $3.4 \pm 0.3$ & $4.0 \pm 0.3$ \\
\hline rMSSD (ms) & $5.9 \pm 0.7$ & $4.3 \pm 0.4^{*}$ & $3.7 \pm 0.3$ & $4.2 \pm 0.3$ \\
\hline Total power $\left(\mathrm{ms}^{2}\right)$ & $25.2 \pm 5.1$ & $13.6 \pm 2.4^{*}$ & $12.4 \pm 2.1$ & $14.9 \pm 2.1$ \\
\hline $\mathrm{LF}_{\mathrm{nu}}$ & $10.7 \pm 1.9$ & $7.8 \pm 1.1$ & $10.8 \pm 1.6$ & $10.9 \pm 1.7$ \\
\hline $\mathrm{HF}_{\mathrm{nu}}$ & $84.7 \pm 2.7$ & $83.1 \pm 2.6$ & $83.3 \pm 2.3$ & $83.8 \pm 2.2$ \\
\hline $\mathrm{LF} / \mathrm{HF}$ & $0.16 \pm 0.03$ & $0.1 \pm 0.01$ & $0.13 \pm 0.02$ & $0.14 \pm 0.02$ \\
\hline $\mathrm{dP} / \mathrm{dt}_{\max }(\mathrm{mm} \mathrm{Hg} / \mathrm{s})$ & $2195 \pm 250$ & $2498 \pm 299$ & $2481 \pm 233$ & $2410 \pm 313$ \\
\hline
\end{tabular}

$\mathrm{dP} / \mathrm{dtmax}$, maximum rate of rise in left ventricular pressure.

${ }^{*} P<0.05$ vs. corresponding non-PE values in the same rat sex.

offspring, reflex bradycardic responses were significantly attenuated by LPS in PE rats only (Fig. 6C), but a depressant action of LPS on tachycardic reflexes was observed in both PE and non-PE female offspring (Fig. 6D). Additionally, comparisons of intersex values showed that BRS in LPS-treated non-PE male offspring was significantly lower than respective values in female offspring for phenylephrine $(1.2 \pm 0.05$ vs. $1.66 \pm 0.17$ beats $/ \mathrm{min}$ per millimeters of $\mathrm{Hg}, P<0.05)$ as well as sodium nitroprusside $(0.92$ \pm 0.13 vs. $1.52 \pm 0.18$ beats $/ \mathrm{min}$ per millimeters of $\mathrm{Hg}, P<0.05$ ). By contrast, no sex differences were observed in BRS values of LPS-treated male and female offspring of PE mothers (Fig. 6).

Pre-eclampsia Sensitizes Male Offspring to Endotoxic Myocardial and Brainstem Inflammation. The effects of LPS on immunohistochemical protein expressions of TLR-4 and MCP-1 in cardiac and brainstem tissues of the offspring of PE or control (non-PE) mothers are depicted in Figs. 7-9. No sex differences were observed in the expression of either protein in all tissues of control offspring. A clear evidence of upregulated TLR-4 expression in cardiac and neuronal pools of NTS and RVLM was noted in LPS-treated offspring of non-PE rats of the two sexes compared with respective control values in non-PE offspring. These inflammatory responses to LPS in cardiac and neuronal tissues were exacerbated in male, but not female, PE offspring rats (Figs. 7A, 8A, and 9A).

Alternatively, immunohistochemical analysis of MCP-1 expression revealed site- and sex-specific effects. Compared with saline treatment of non-PE males, LPS caused significant elevations in MCP-1 expression in cardiac and neuronal tissues of (Figs. 7B, 8B, and 9B). In this circumstance, the treatment of PE male offspring with LPS resulted in significantly higher expression levels of MCP-1 in cardiac, but not neuronal, tissues. In LPS-treated female offspring of non-PE mothers, elevated MCP-1 expression was observed in cardiac, but not NTS or RVLM, tissues, and these effects of LPS remained unaltered in $\mathrm{PE}$ female offspring (Figs. 7B, 8B, and 9B). ELISA determinations showed that serum MCP-1 was
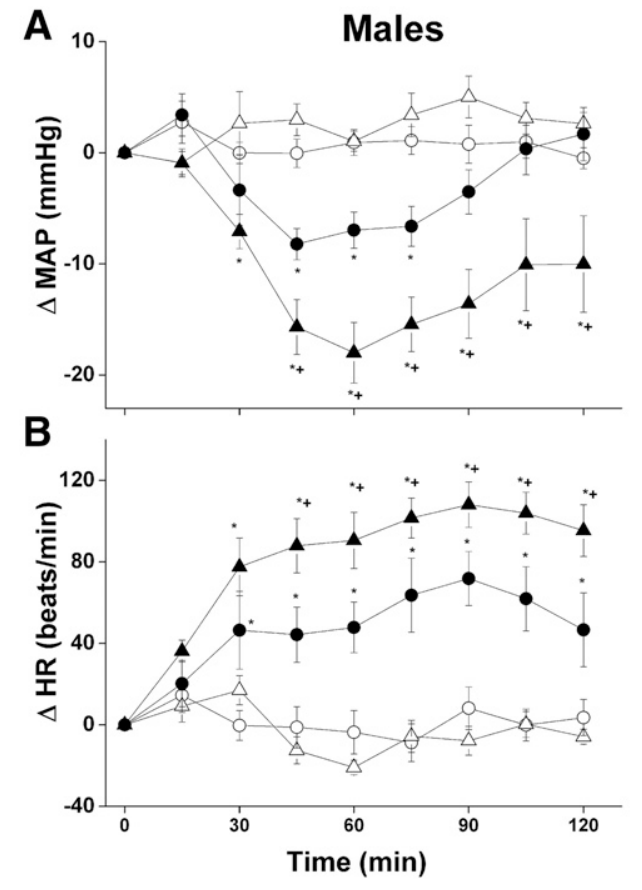

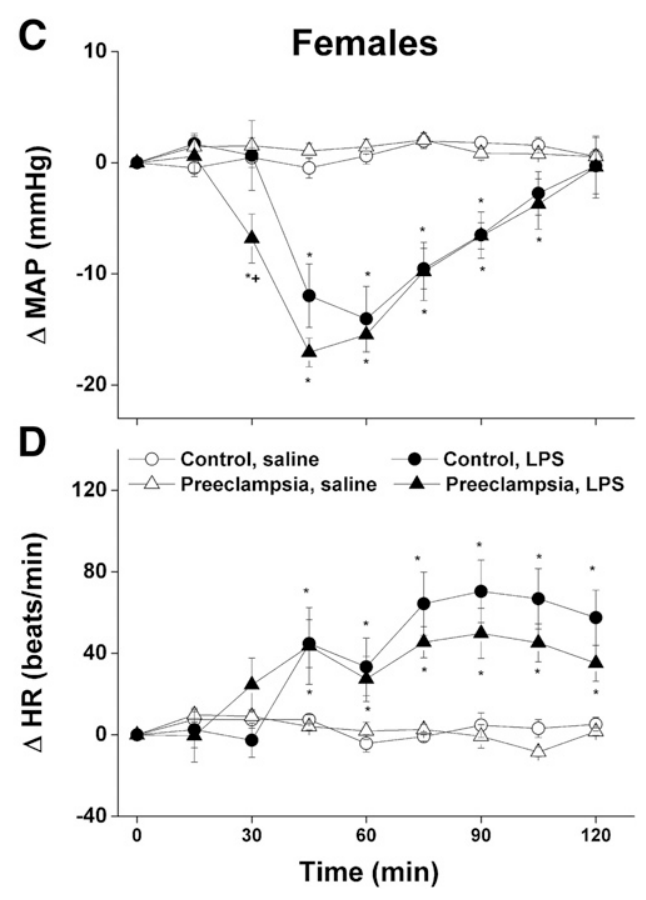

Fig. 2. Effect of pre-eclamsia on changes in MAP (panels A and C) and HR (panels $\mathrm{B}$ and $\mathrm{D})$ evoked by intravenous LPS $(5 \mathrm{mg} / \mathrm{kg})$ or equal volume of saline in adult male and female offspring rats. Values are means \pm S.E.M. of eight observations. The repeated measures ANOVA followed by the Tukey's post hoc test was employed to measure statistical significance. $* P<0.05$ vs. respective "control, saline" values, ${ }^{+} P<0.05$ vs. respective "control, LPS" values in the same rat sex. 

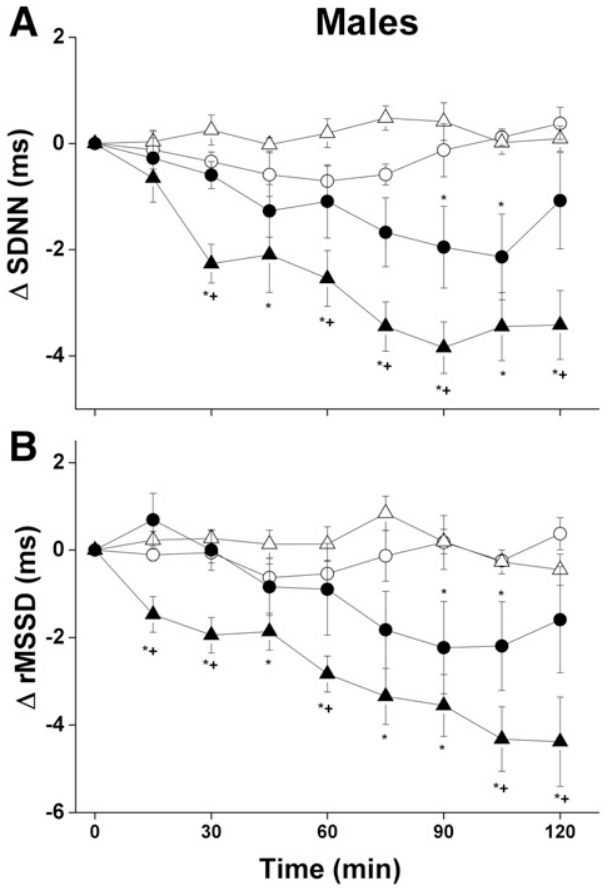
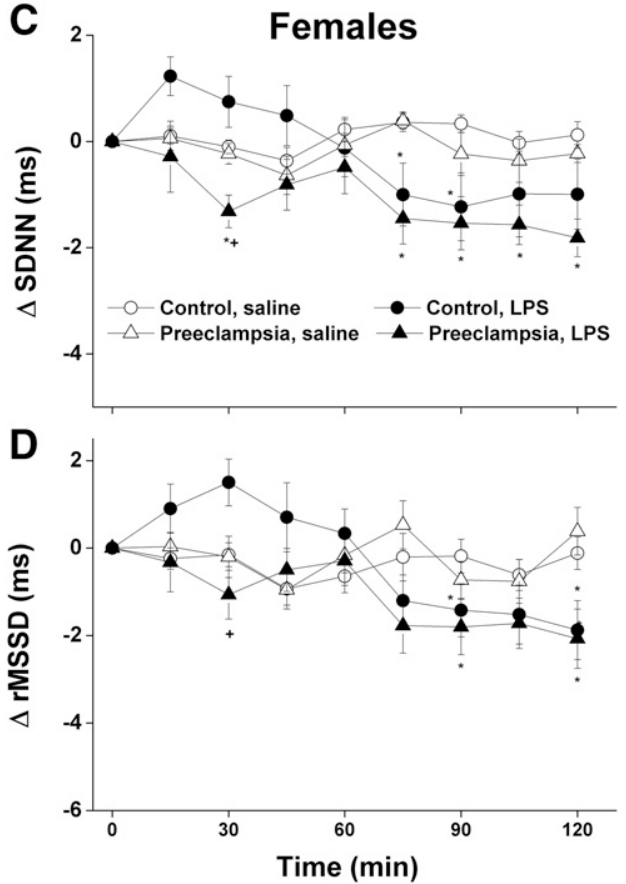

Fig. 3. Effect of pre-eclampsia on changes in time-domain indices of heart rate variability (SDNN, panels A and C; rMSSD, panels $\mathrm{B}$ and $\mathrm{D}$ ) evoked by intravenous LPS $(5 \mathrm{mg} / \mathrm{kg})$ or equal volume of saline in adult male and female offspring rats. Values are means \pm S.E.M. of eight observations. The repeated measures ANOVA followed by the Tukey's post hoc test was employed to measure statistical significance. $* P<0.05$ vs. respective "control, saline" values, ${ }^{+} P<0.05$ vs. respective "control, LPS" values in the same rat sex. increased after LPS challenge in non-PE offspring, and this effect was potentiated in the two sexes of $\mathrm{PE}$ offspring (Fig. 7C). Generally, rises in serum MCP-1 by LPS were more noted in male compared with female offspring of non-PE (99 \pm 3 vs. $78 \pm 3 \mathrm{pg} / \mathrm{ml}, P<0.05$ ) or PE mothers $(160 \pm 6$ vs. $137 \pm 5$ pg/ml, $P<0.05$ ) (Fig. 7C).

\section{Discussion}

This study is the first to report on sex specificity of PE fetal programming on inflammatory and cardiovascular effects of endotoxemia in adult rat offspring. Compared with non-PE counterparts, LPS treatment of male offspring of PE mothers exhibited greater 1) falls in BP and time and frequency indices of cardiac autonomic function, 2) rises in $\mathrm{HR}$ and cardiac contractility, and 3) increases in abundance of inflammatory cytokines in cardiac brainstem tissues. Because none of these exaggerated effects were noted in female offspring, it is concluded that fetal programming to $\mathrm{PE}$-mediated intrauterine adaptations primes male offspring toward endotoxic sequels in adulthood.

The development of $\mathrm{PE}$ in current study was verified by rises in SBP and proteinuria. Notably, the adverse implications of PE
A
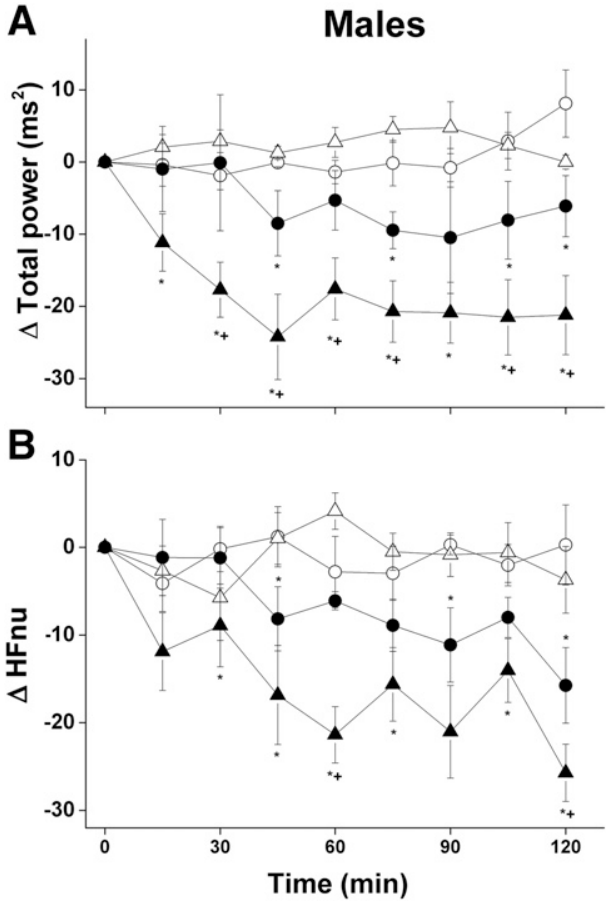

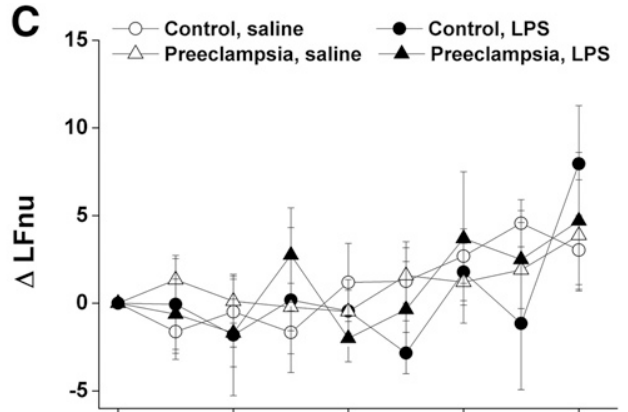

D

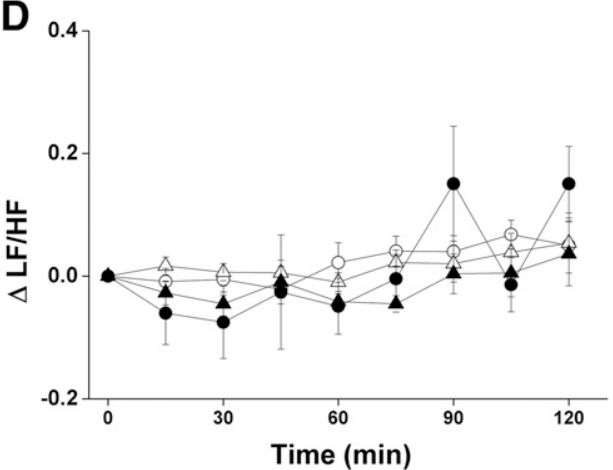

Fig. 4. Effect of pre-eclampsia on changes in frequency-domain indices of heart rate variability (total power, panel $\mathrm{A} ; \mathrm{HF}_{\text {nu,panel } \mathrm{B}}$ $\mathrm{LF}_{\mathrm{nu}}$, panel C; and LF:HF, panel D) evoked by intravenous LPS $(5 \mathrm{mg} / \mathrm{kg})$ or equal volume of saline in adult male and female offspring rats. Values are means \pm S.E.M. of eight observations. The repeated measures ANOVA followed by the Tukey's post hoc test was employed to measure statistical significance. ${ }^{*} P<0.05$ vs. respective "control, saline" values, ${ }^{+} P<0.05$ vs. respective "control, LPS" values in the same rat sex. 

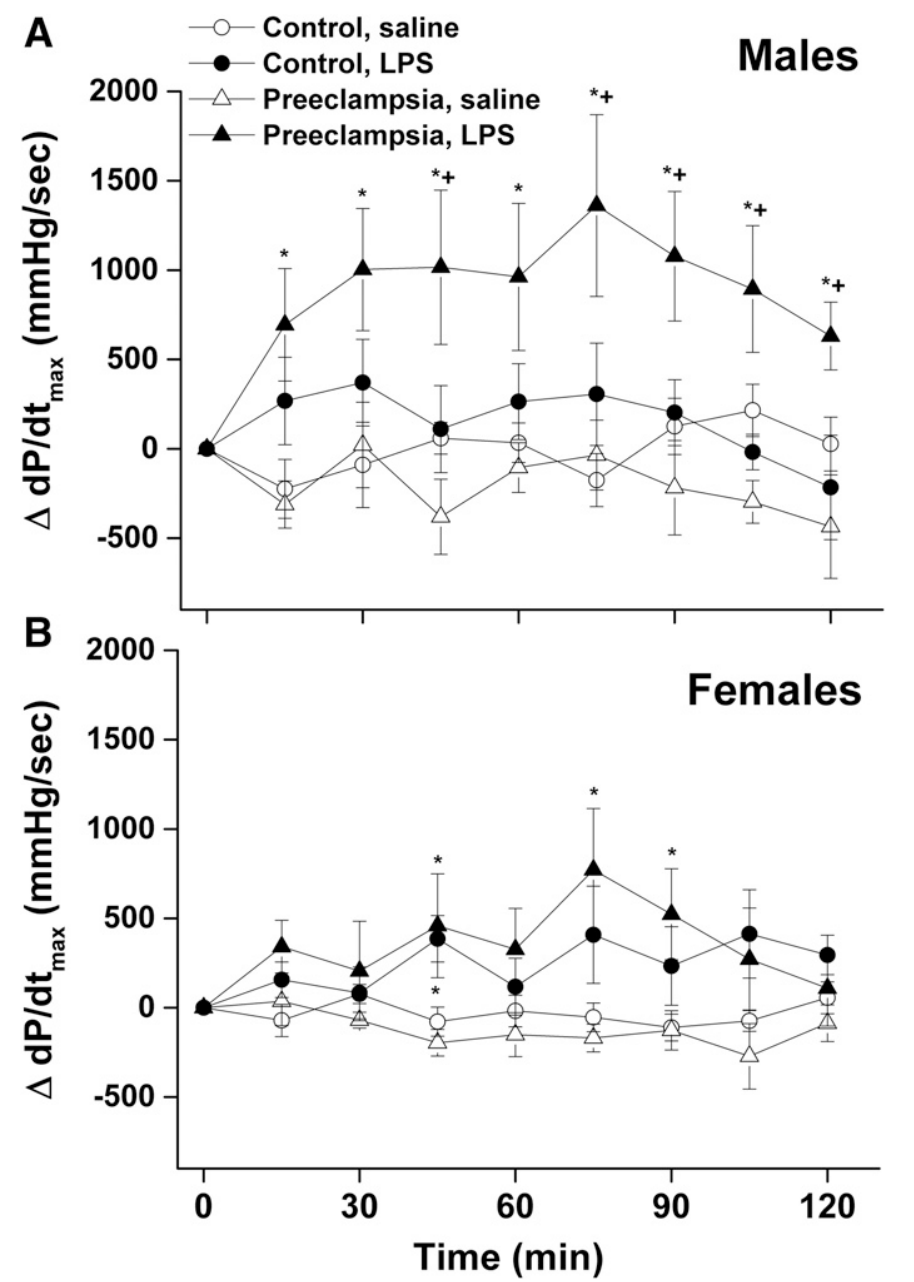

Fig. 5. Effect of pre-eclampsia on changes in the maximum rate of rise of blood pressure waves (dP/dtmax, correlate of left ventricular contractility) evoked by intravenous LPS $(5 \mathrm{mg} / \mathrm{kg})$ or equal volume of saline in adult male (panel A) and female (panel B) offspring rats. Values are means \pm S.E.M. of eight observations. The repeated measures ANOVA followed by the Tukey's post hoc test was employed to measure statistical significance. $* P<0.05$ vs. respective "control, saline" values, ${ }^{+} P<0.05$ vs. respective "control, LPS" values in the same rat sex.

go beyond the gestational period and are likely to impose longterm and possibly sexually related health influences in offspring. For instance, whereas sex-independent metabolic derangements were observed in mouse offspring born to $\mathrm{PE}$ mothers treated with soluble fms-like tyrosine kinase-1, hypertension appeared in male offspring only (Lu et al., 2007; Bytautiene et al., 2011). Fetal undernutrition induced by placental insufficiency during late gestation causes hypertension and mesenteric artery dysfunction in two consecutive generations of male and female offspring (Anderson et al., 2006). Our results demonstrated higher SBP and proteinuria in $\mathrm{PE}$ male, but not female, offspring suggesting preferential male vulnerability to fetal programming in L-NAME PE model. Together, PE insult type and gestational intervention time play key roles in identifying fetal programming profile and aftereffects in offspring of the two sexes.

The present study reveals three important observations that reinforce the advantageous cardiovascular state of female offspring of PE mothers against endotoxemia. First, although LPS caused hypotension and tachycardia in the two sexes of
non-PE rat offspring, HRV signs of cardiac autonomic dysfunction such as reduced overall cardiac autonomic balance (SDNN and total power) and parasympathetic (rMSSD and $\mathrm{HF}$ ), but not sympathetic (LF), activity were evident only in male offspring. These findings are consistent with greater disturbances in respiratory function observed in male sex such as pulmonary inflammation and hyperventilation, which are believed to be modulated by cardiac vagal function (Tang et al., 1998; Kosyreva et al., 2012; Perez-Quilis et al., 2017). In this context, the reason for the unaltered spectral index of cardiac sympathovagal balance (LF:HF) is not clear. This may relate to the view that in addition to sympathetic drive, other factors including vagal control, sex, and age could contribute to LF component of HRV (Stein et al., 1994; Sgoifo et al., 1997; Parati et al., 2006). Likewise, vagal activity is not entirely responsible for HF oscillations of HRV. Residual HF oscillations observed after autonomic blockade or cardiac transplantation are caused by mechanical respiratory modulation of sinus node (Bernardi et al., 1989).

Second, whereas hypotensive and HRV depressant actions of LPS were remarkably exaggerated in male offspring of PE
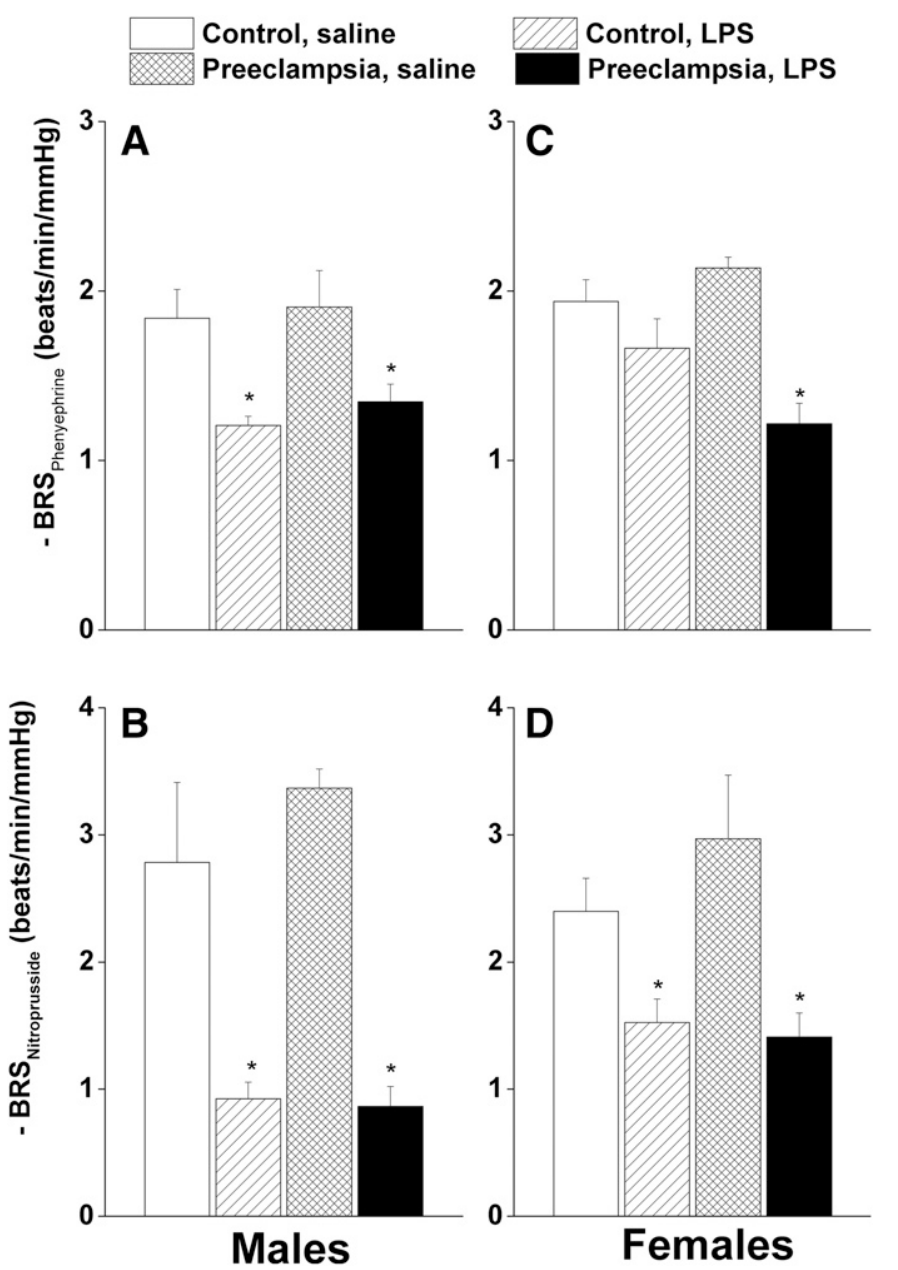

Fig. 6. Effect of pre-eclampsia on $\mathrm{BRS}_{\text {phenylephrine }}$ (panels $\mathrm{A}$ and $\mathrm{C}$ ) and $\mathrm{BRS}_{\text {nitroprusside }}$ (panels B and D) in adult male and female offspring rats treated with intravenous LPS $(5 \mathrm{mg} / \mathrm{kg}$ ) or equal volume of saline. Values are means \pm S.E.M. of eight observations. The repeated measures ANOVA followed by the Tukey's post hoc test was employed to measure statistical significance. $* P<0.05$ vs. respective non-pre-eclamptic "control, saline" values. 


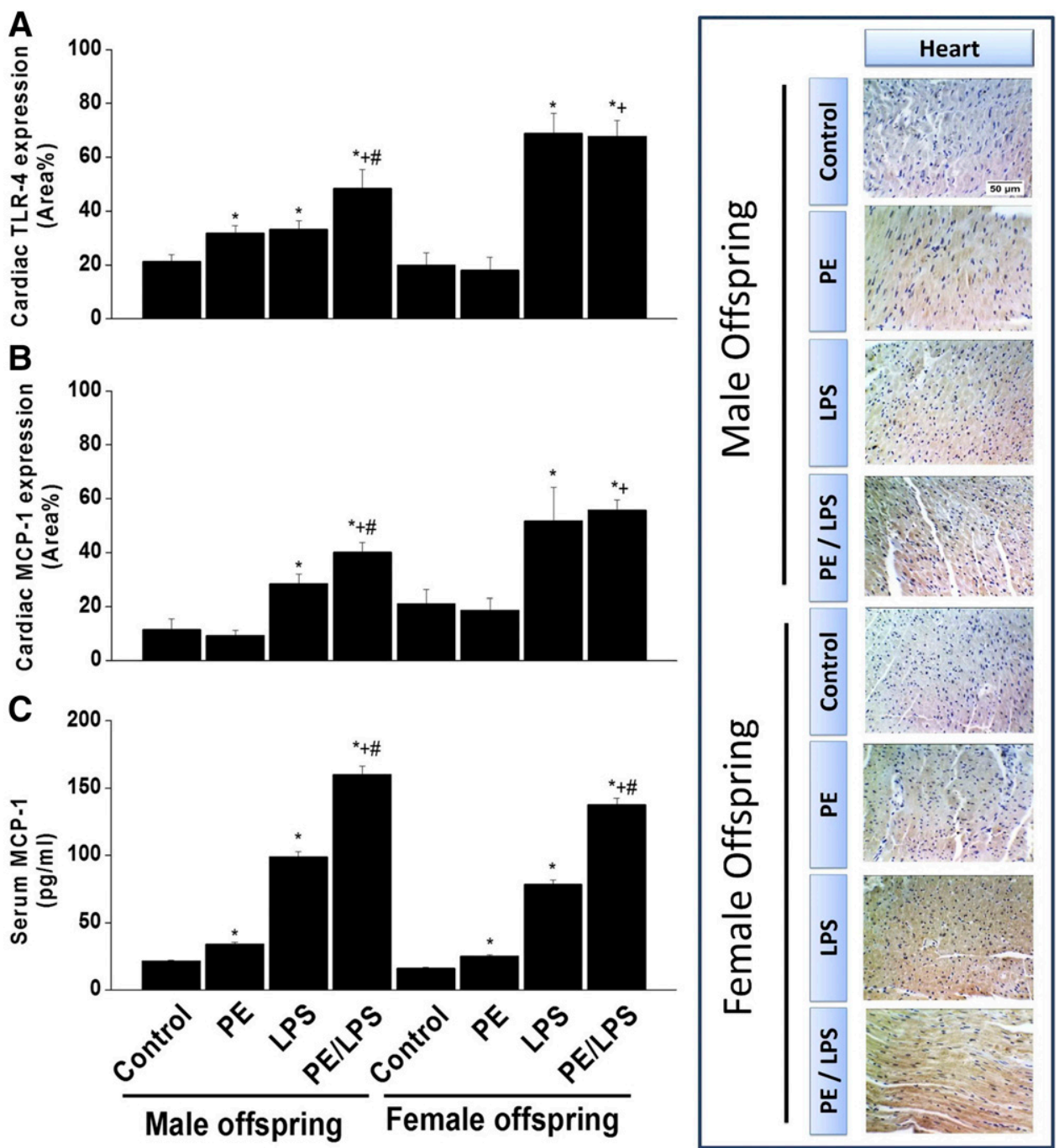

Fig. 7. Effect of $\mathrm{PE}$ on changes in serum MCP-1 (panel C) and cardiac immunohistochemical protein expression of TLR-4 (panel A) and MCP-1 (panel B) evoked by intravenous LPS $(5 \mathrm{mg} / \mathrm{kg})$ in adult male and female offspring rats. Representative images for immunostained sections from the heart are shown. Values are means \pm S.E.M. of five observations. The one-way ANOVA followed by the Tukey's post hoc test was employed to measure statistical significance. $* P<0.05$ vs. respective "control" values, ${ }^{+} P<0.05$ vs. respective "PE" values, ${ }^{\#} P<0.05$ vs. respective "LPS" values in the same rat sex.

rats, the privileged female status remained a consistent feature in LPS-treated female offspring of PE mothers because no intensification of LPS hypotension was observed and little or no changes in HRV indices occurred. Considering the significantly greater BP falls caused by LPS in female compared with male offspring of non-PE rats, the lack of potentiated LPS hypotension in female offspring of PE rats might be explained, in part, by the pre-existing dampening of compensatory mechanisms. The latter are functional and molecular ramifications that are activated to offset undesirable effects of endotoxemia (Yamaguchi et al., 2006). Third, male, but not female, endotoxic offspring exhibited dramatic rises in myocardial contractility correlate (dP/dtmax), which might serve an adaptive response to weakened cardiomotor vagal input in males. Considering that altered cardiac autonomic and contractility functions often predispose to cardiovascular disturbances and accelerated mortality in endotoxemia (Klöckner et al., 2011; Mazzeo et al., 2011; Sallam et al., 2018), the preservation of such traits in female offspring infers their resistance to harsh intrauterine environment featured during $\mathrm{PE}$.

Contradictory information is available regarding whether baroreflex changes are causally related to other hemodynamic actions of endotoxemia (Bigger et al., 1989; Vayssettes-
Courchay et al., 2005). In this study, baroreceptor assessment by vasoactive method unraveled data that were not exactly analogous to those of hemodynamic or HRV investigations. The attenuated reflex bradycardic ( $\left.\mathrm{BRS}_{\text {phenylephrine }}\right)$ and tachycardic $\left(\mathrm{BRS}_{\text {nitroprusside }}\right)$ responses in endotoxic male offspring of non-PE mothers was not accentuated in male counterparts from PE mothers. Alternatively, reflex bradycardia was reduced by LPS in female offspring of PE, but not non-PE, mothers. Because reflex bradycardia and tachycardia are predominantly mediated through vagal and sympathetic activity, respectively (El-Mas et al., 2002; La Rovere and Christensen, 2015), current data indicate that unlike HRV, PE unveiled LPS attenuation of reflex cardiomotor vagal activity in female offspring. More studies are warranted to define the precise role of cardiac neural control in PE fetal programming of endotoxemia.

Inflammatory signals in peripheral and central tissues participate in endotoxic organ damage (Dantzer, 2009; Cazareth et al., 2014). Peripheral inflammatory signals enter the brain through NTS, which arbitrates, along with its polysynaptic medullary and hypothalamic connections, cardiovascular irregularities of endotoxemia (Lin et al., 1999; Pavlov et al., 2003; Sirivelu et al., 2012). Recently, we reported that upregulation of brainstem neuroinflammatory pathways of 

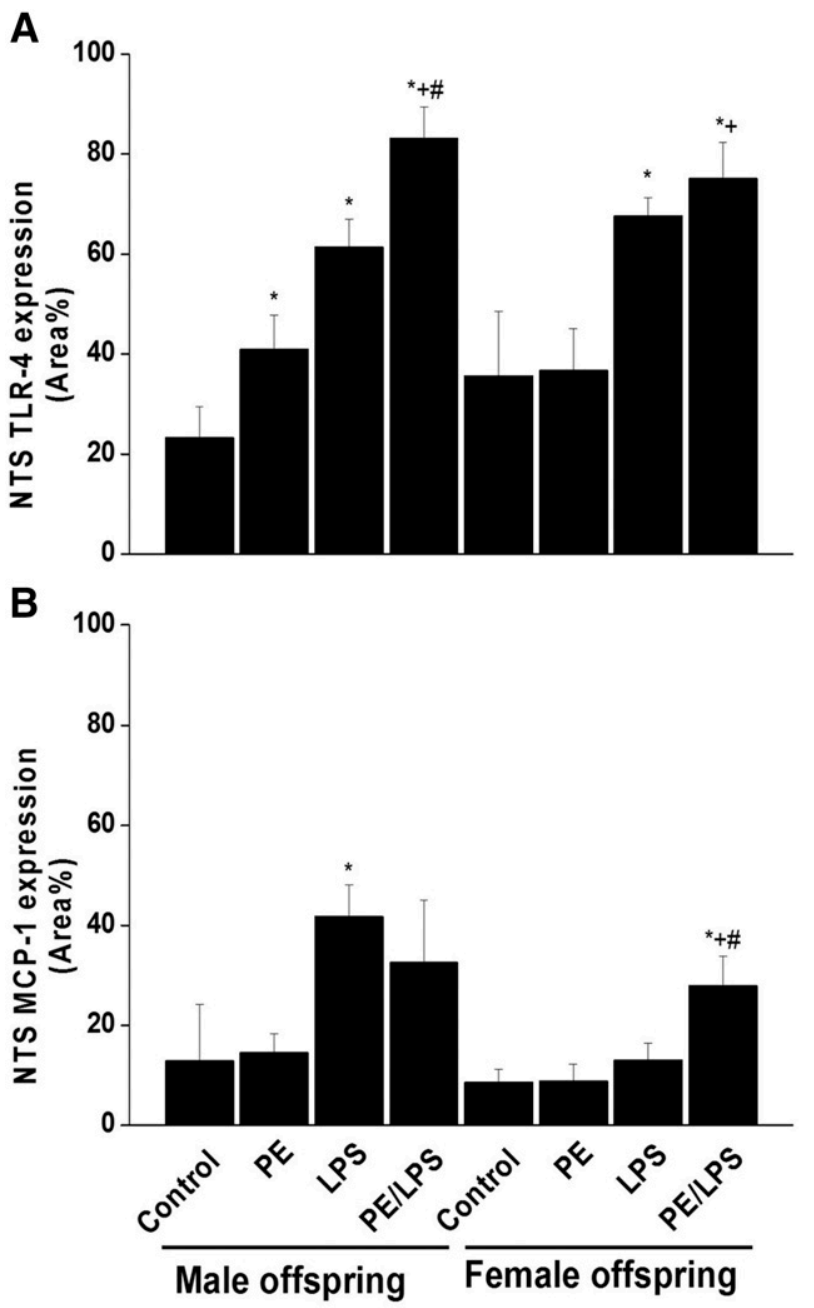

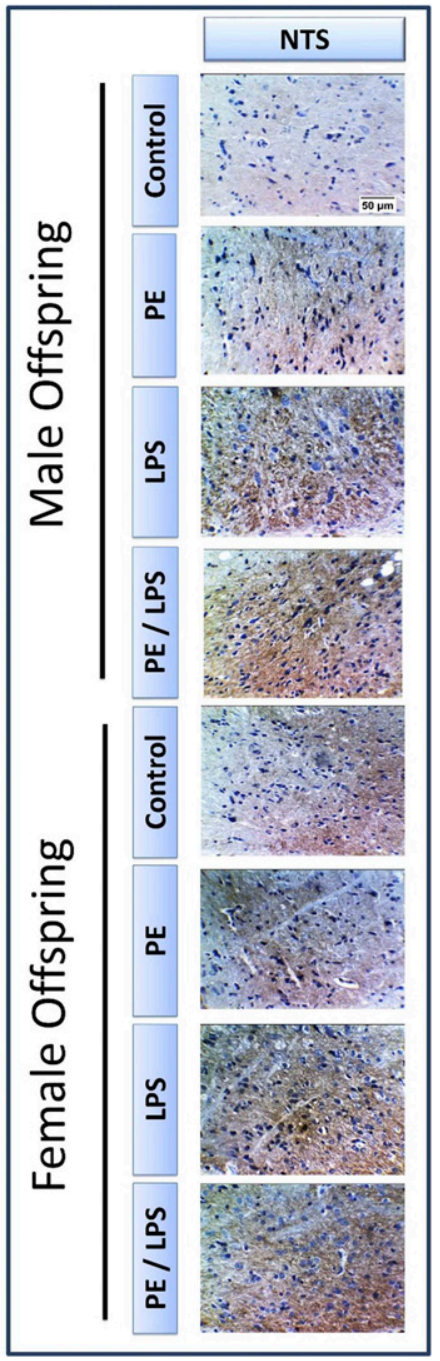

Fig. 8. Effect of $\mathrm{PE}$ on changes in immunohistochemical protein expression of TLR-4 (panel A) and MCP-1 (panel B) in NTS evoked by intravenous LPS ( $5 \mathrm{mg} / \mathrm{kg}$ ) in adult male and female offspring rats. Representative images for immunostained sections from the NTS are shown. Values are means \pm S.E.M. of five observations. The one-way ANOVA followed by the Tukey's post hoc test was employed to measure statistical significance. $* P<0.05$ vs. respective "control" values, ${ }^{+} P<0.05$ vs. respective "PE" values, ${ }^{\#} P<0.05$ vs. respective "LPS" values in the same rat sex.
$\mathrm{NF} \kappa \mathrm{B}$ mediates endotoxic hypotension and autonomic depression (Sallam et al., 2019). The current study implicates brainstem neuroanatomical pools of TLR-4 in PE-related fetal programming that worsened endotoxic cardiovascular manifestations in adult male, but not female, offspring. This is supported by the findings that exacerbated reductions in BP and HRV by endotoxemia in male progeny of PE mothers were paralleled with potentiated TLR-4 expression in heart and brainstem pools of NTS and RVLM. Sex specificity of this interaction was corroborated by the lack of such intensified effects in female offspring of same litters. Increased placental TLR4 expression is also seen in PE induced by the single administration of a very low dose of LPS early during pregnancy (Xue et al., 2015). TLR-4 is a key recognition receptor that serves as principal driver of host inflammatory response to bacterial infections (Iwasaki and Medzhitov, 2004; Fink, 2014). TLR4 upregulation by LPS triggers downstream activation of IкB kinase and NF- $\kappa$ B and subsequent generation of a wide array of cytokines, chemokines ( $\mathrm{Li}$ et al., 2017; Lee et al., 2019). These molecules mediate LPSinduced cardiac mitochondrial dysfunction and apoptosis (Tien et al., 2010) and positively correlate with worsened clinical outcomes and mortality (Akira and Takeda, 2004; Liu and Malik, 2006).
MCP-1, a potent chemotactic factor for monocytes, is generated after LPS binding to TLR-4 and provokes systemic inflammation and organ damage (Zhu et al., 2017). Our data showed that MCP-1 expression profile mimicked that of TLR-4 in cardiac, but not neuronal, tissues. In heart, the increased abundance of MCP-1 in response to LPS challenge was exaggerated in male, but not female, offspring of PE mothers. By contrast, brainstem nuclei of male offspring of PE rats exhibited upregulated MCP-1 expression, but no further increments were observed in same rats after LPS treatment. These results suggest preferential involvement of cardiac, but not neuronal, MCP-1 in heightened endotoxic hypotension and cardiac autonomic depression in male offspring.

One potential limitation of current study is the use of $\mathrm{dP} /$ $\mathrm{dt}_{\max }$ as a measure of cardiac contractility. The LabChart-7 pro software adopted here allowed computation of maximum rate of pressure rise across $\mathrm{BP}$ wave form $\left(\mathrm{dP} / \mathrm{dt}_{\text {max }}\right)$, which serves as an indirect measure of cardiac contractile force (Mehta et al., 1998). The validity of arterial $\mathrm{dP} / \mathrm{dt}_{\max }$ as an index of left ventricular contractility remains questionable because it is derived from arterial pressure measured with a fluid-filled catheter and is influenced by preload and vascular filling conditions. Cardiac function is typically 

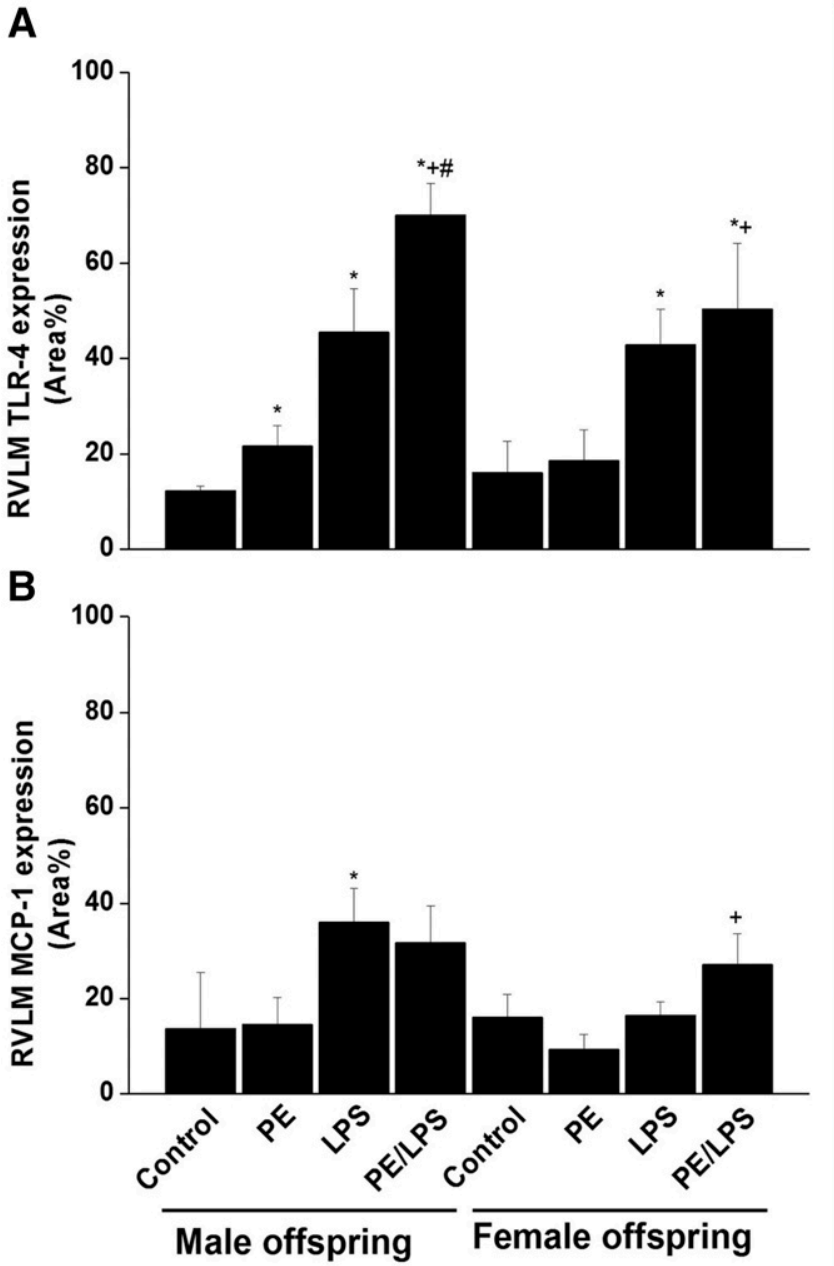

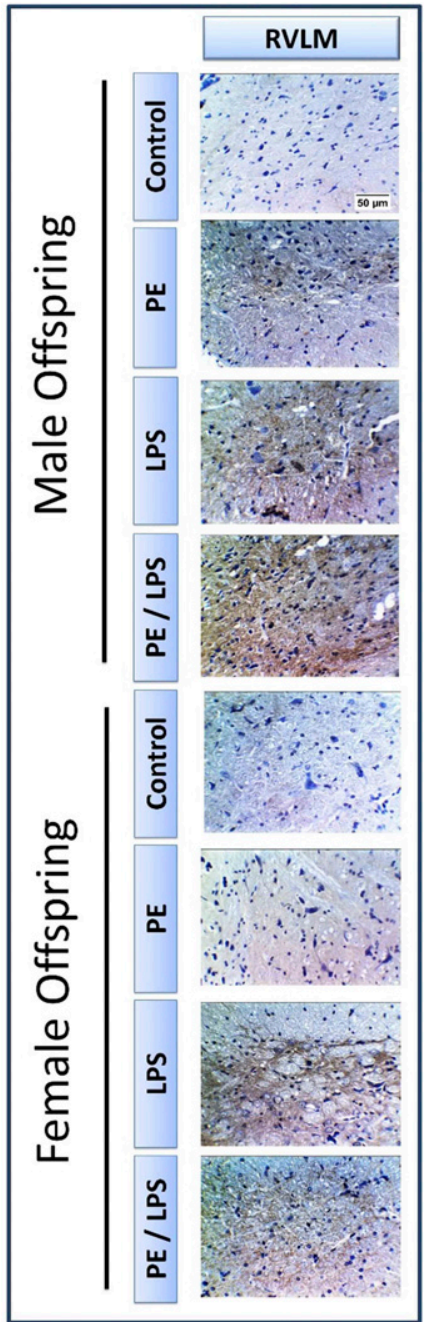

Fig. 9. Effect of $\mathrm{PE}$ on immunohistochemical protein expression of TLR-4 (panel A) and MCP-1 (panel B) in the RVLM evoked by intravenous LPS in conscious offspring. Representative images for immunostained sections from the RVLM are shown. Values are means \pm S.E.M. of five observations. The one-way ANOVA followed by the Tukey's post hoc test was employed to measure statistical significance. $* P<0.05$ vs. respective "control" values, ${ }^{+} P<0.05$ vs. respective "PE" values, ${ }^{\#} P<0.05$ vs. respective "LPS" values in the same rat sex. measured by electrocardiographic or echocardiographic techniques (Carroll et al., 2006; Adeyemi et al., 2009). Nevertheless, some studies have established significant correlation between arterial dP/dtmax and end-systolic elastance, the gold standard measure of LV contractility, during hemodynamic perturbations including endotoxemia (Morimont et al., 2012).

Taken together, current molecular and neuroanatomical evidence suggests possible roles for PE-induced fetal programming in offspring predisposition to health hazards induced by endotoxemia in adult life. PE amplifies endotoxic manifestations of hypotension, tachycardia, and cardiac autonomic dysfunction in male offspring via exacerbating myocardial and neuroinflammatory pathways of the NTS and RVLM. The lack of such injurious states in female littermates suggests sexual dimorphism in the capacity of PE fetal programming to shape endotoxic outcomes.

\section{Authorship Contributions}

Participated in research design: El-Gowilly, Helmy, El-Mas.

Conducted experiments: Abuiessa, Wedn.

Performed data analysis: Abuiessa, Wedn, El-Gowilly, Helmy, ElMas.

Wrote or contributed to the writing of the manuscript: Abuiessa, Wedn, El-Gowilly, Helmy, El-Mas.

\section{References}

Adeyemi O, Roberts S, Harris J, West H, Shome S, and Dewhurst M (2009) QA interval as an indirect measure of cardiac contractility in the conscious telemeterised rat: model optimisation and evaluation. J Pharmacol Toxicol Methods 60:159-166.

Akira S and Takeda K (2004) Toll-like receptor signalling. Nat Rev Immunol 4: 499-511.

Amaral TAS, Ognibene DT, Carvalho LCRM, Rocha APM, Costa CA, Moura RS, and Resende AC (2018) Differential responses of mesenteric arterial bed to vasoactive substances in L-NAME-induced preeclampsia: role of oxidative stress and endothelial dysfunction. Clin Exp Hypertens 40:126-135.

Anderson CM, Lopez F, Zimmer A, and Benoit JN (2006) Placental insufficiency leads to developmental hypertension and mesenteric artery dysfunction in two generations of Sprague-Dawley rat offspring. Biol Reprod 74:538-544.

Bernardi L, Keller F, Sanders M, Reddy PS, Griffith B, Meno F, and Pinsky MR (1989) Respiratory sinus arrhythmia in the denervated human heart. J Appl Physiol (1985) 67:1447-1455.

Bigger JT Jr., La Rovere MT, Steinman RC, Fleiss JL, Rottman JN, Rolnitzky LM, and Schwartz PJ (1989) Comparison of baroreflex sensitivity and heart period variability after myocardial infarction. $J$ Am Coll Cardiol 14:1511-1518.

Brown MA, Magee LA, Kenny LC, Karumanchi SA, McCarthy FP, Saito S, Hall DR, Warren CE, Adoyi G, and Ishaku S; International Society for the Study of Hypertension in Pregnancy (ISSHP) (2018) Hypertensive disorders of pregnancy: ISSHP classification, diagnosis, and management recommendations for international practice. Hypertension 72:24-43.

Bytautiene E, Tamayo E, Kechichian T, Drever N, Gamble P, Hankins GD, and Saade GR (2011) Prepregnancy obesity and sFlt1-induced preeclampsia in mice: developmental programming model of metabolic syndrome. Am J Obstet Gynecol 204:398.e1-398.e8.

Carroll JF, Zenebe WJ, and Strange TB (2006) Cardiovascular function in a rat model of diet-induced obesity. Hypertension 48:65-72.

Cazareth J, Guyon A, Heurteaux C, Chabry J, and Petit-Paitel A (2014) Molecular and cellular neuroinflammatory status of mouse brain after systemic lipopolysaccharide challenge: importance of CCR2/CCL2 signaling. J Neuroinflammation 11:132.

Chen Y, Xue F, Han C, Yang H, Han L, Li K, Li J, Xu Q, Li Z, Yuan B, et al. (2019) Ferulic acid ameliorated placental inflammation and apoptosis in rat with preeclampsia. Clin Exp Hypertens 41:524-530. 
Dantzer R (2009) Cytokine, sickness behavior, and depression. Immunol Allergy Clin North Am 29:247-264.

Davis EF, Lazdam M, Lewandowski AJ, Worton SA, Kelly B, Kenworthy Y, Adwani S, Wilkinson AR, McCormick K, Sargent I, et al. (2012) Cardiovascular risk factors in children and young adults born to preeclamptic pregnancies: a systematic review. Pediatrics 129:e1552-e1561.

Dickson K and Lehmann C (2019) Inflammatory response to different toxins in experimental sepsis models. Int J Mol Sci 20:(18). pii: E4341.

Ding X, Yang Z, Han Y, and Yu H (2015) Correlation of long-chain fatty acid oxidation with oxidative stress and inflammation in pre-eclampsia-like mouse models. Placenta 36:1442-1449.

El-Lakany MA, Fouda MA, El-Gowelli HM, El-Gowilly SM, and El-Mas MM (2018) Gonadal hormone receptors underlie the resistance of female rats to inflammatory and cardiovascular complications of endotoxemia. Eur J Pharmacol 823:41-48.

el-Mas MM, Abdel-Galil AG, el-Gowelli HM, and Daabees TT (1997) Short-term aortic barodenervation diminishes alpha 1-adrenoceptor reactivity in rat aortic smooth muscle. Eur J Pharmacol 322:201-210.

El-Mas MM and Abdel-Rahman AA (1999) Role of the sympathetic control of vascular resistance in ethanol-clonidine hemodynamic interaction in SHRs. $J$ Cardiovasc Pharmacol 34:589-596.

El-Mas MM and Abdel-Rahman AA (2007) Intermittent clonidine regimen abolishes tolerance to its antihypertensive effect: a spectral study. J Cardiovasc Pharmacol 49:174-181.

El-Mas MM and Abdel-Rahman AA (2013) Cardiovascular autonomic modulation by nitric oxide synthases accounts for the augmented enalapril-evoked hypotension in ethanol-fed female rats. Alcohol 47:339-346.

El-Mas MM, Afify EA, Omar AG, and Sharabi FM (2002) Cyclosporine attenuates the autonomic modulation of reflex chronotropic responses in conscious rats. Can $J$ Physiol Pharmacol 80:766-776.

El-Mas MM, El-Gowelli HM, Ghazal AR, Harraz OF, and Mohy El-Din MM (2009) Facilitation of central imidazoline I(1)-site/extracellular signal-regulated kinase/p38 mitogen-activated protein kinase signalling mediates the hypotensive effect of ethanol in rats with acute renal failure. $\mathrm{Br} J$ Pharmacol 158: 1629-1640.

El-Mas MM, Helmy MW, Ali RM, and El-Gowelli HM (2015) Celecoxib, but not indomethacin, ameliorates the hypertensive and perivascular fibrotic actions of cyclosporine in rats: role of endothelin signaling. Toxicol Appl Pharmacol 284:1-7.

El-Mas MM, Mohy El-Din MM, Helmy MM, and Omar AG (2012) Redox imbalances incite the hypertensive, baroreflex, and autonomic effects of cyclosporine in rats. Eur J Pharmacol 694:82-88.

El-Mas MM, Zhang J, and Abdel-Rahman AA (2006) Upregulation of vascular inducible nitric oxide synthase mediates the hypotensive effect of ethanol in conscious female rats. J Appl Physiol (1985) 100:1011-1018.

Faas MM, Schuiling GA, Baller JF, Visscher CA, and Bakker WW (1994) A new animal model for human preeclampsia: ultra-low-dose endotoxin infusion in pregnant rats. Am J Obstet Gynecol 171:158-164.

Fink MP (2014) Animal models of sepsis. Virulence 5:143-153.

Flierl MA, Rittirsch D, Huber-Lang MS, Sarma JV, and Ward PA (2008) Molecular events in the cardiomyopathy of sepsis. Mol Med 14:327-336.

Fox R, Kitt J, Leeson P, Aye CYL, and Lewandowski AJ (2019) Preeclampsia: risk factors, diagnosis, management, and the cardiovascular impact on the offspring. $J$ Clin Med 8:(10). pii: E1625..

Godfrey KM and Barker DJ (2001) Fetal programming and adult health. Public Health Nutr 4:611-624.

Haddad B, Deis S, Goffinet F, Paniel BJ, Cabrol D, and Siba BM (2004) Maternal and perinatal outcomes during expectant management of 239 severe preeclamptic women between 24 and 33 weeks' gestation. Am J Obstet Gynecol 190:1590-1595 discussion 1595-1597.

Han YW, Yang Z, Ding XY, and Yu H (2015) Differences in liver injury and trophoblastic mitochondrial damage in different preeclampsia-like mouse models. Chin Med J (Engl) 128:1627-1635.

Helmy MW, El-Gowelli HM, Ali RM, and El-Mas MM (2015) Endothelin ETA receptor/lipid peroxides/COX-2/TGF- $\beta 1$ signalling underlies aggravated nephrotoxicity caused by cyclosporine plus indomethacin in rats. $\mathrm{Br} J$ Pharmacol 172 $4291-4302$.

Igosheva N, Klimova O, Anishchenko T, and Glover V (2004) Prenatal stress alters cardiovascular responses in adult rats. J Physiol 557:273-285.

Iwasaki A and Medzhitov R (2004) Toll-like receptor control of the adaptive immune responses. Nat Immunol 5:987-995.

Klein SL and Flanagan KL (2016) Sex differences in immune responses. Nat Rev Immunol 16:626-638.

Klöckner U, Rueckschloss U, Grossmann C, Ebelt H, Müller-Werdan U, Loppnow H, Werdan K, and Gekle M (2011) Differential reduction of HCN channel activity by various types of lipopolysaccharide. J Mol Cell Cardiol 51:226-235.

Kosyreva AM, Simonova EY, and Makarova OV (2012) Gender differences in pulmonary and immune response in acute experimental endotoxicosis. Bull Exp Biol Med 153:340-342.

La Rovere MT and Christensen JH (2015) The autonomic nervous system and cardiovascular disease: role of n-3 PUFAs. Vascul Pharmacol 71:1-10.

Lee S-Y, Hsin L-W, Su M-J, ChangChien C-C, and Ku H-C (2019) A novel isoquinoline derivative exhibits anti-inflammatory properties and improves the outcomes of endotoxemia. Pharmacol Rep 71:1281-1288.

Li J, Zhai Y, Ao L, Hui H, Fullerton DA, Dinarello CA, and Meng X (2017) Interleukin-37 suppresses the inflammatory response to protect cardiac function in old endotoxemic mice. Cytokine 95:55-63.

Lin HC, Wan FJ, Kang BH, Wu CC, and Tseng CJ (1999) Systemic administration of lipopolysaccharide induces release of nitric oxide and glutamate and c-fos expression in the nucleus tractus solitarii of rats. Hypertension 33:1218-1224.
Liu SF and Malik AB (2006) NF-kappa B activation as a pathological mechanism of septic shock and inflammation. Am J Physiol Lung Cell Mol Physiol 290: L622-L645.

Lu F, Bytautiene E, Tamayo E, Gamble P, Anderson GD, Hankins GD, Longo M, and Saade GR (2007) Gender-specific effect of overexpression of sFlt-1 in pregnant mice on fetal programming of blood pressure in the offspring later in life. $A m$ $J$ Obstet Gynecol 197:418.e1-418 e5.

Lv R, Zhou ZQ, Wu HW, Jin Y, Zhou W, and Xu JG (2006) Hydroxyethyl starch exhibits antiinflammatory effects in the intestines of endotoxemic rats. Anesth Analg 103:149-155.

Madazli R, Yuksel MA, Imamoglu M, Tuten A, Oncul M, Aydin B, and Demirayak G (2014) Comparison of clinical and perinatal outcomes in early- and late-onset preeclampsia. Arch Gynecol Obstet 290:53-57.

Marshall SA, Hannan NJ, Jelinic M, Nguyen TPH, Girling JE, and Parry LJ (2018) Animal models of preeclampsia: translational failings and why. Am J Physiol Regul Integr Comp Physiol 314:R499-R508.

Mazzeo AT, La Monaca E, Di Leo R, Vita G, and Santamaria LB (2011) Heart rate variability: a diagnostic and prognostic tool in anesthesia and intensive care. Acto Anaesthesiol Scand 55:797-811.

McCarthy FP, Kingdom JC, Kenny LC, and Walsh SK (2011) Animal models of preeclampsia; uses and limitations. Placenta 32:413-419.

Mehta MC, Jain AC, and Billie M (1998) Combined effects of alcohol and nicotine on cardiovascular performance in a canine model. $J$ Cardiovasc Pharmacol 31: 930-936.

Morimont P, Lambermont B, Desaive T, Janssen N, Chase G, and D'Orio V (2012) Arterial dP/dtmax accurately reflects left ventricular contractility during shock when adequate vascular filling is achieved. BMC Cardiovasc Disord 12:13

Morton A (2016) Imitators of preeclampsia: a review. Pregnancy Hypertens 6:1-9.

Omar AG and El-Mas MM (2004) Time-domain evaluation of cyclosporine interaction with hemodynamic variability in rats. Cardiovasc Drugs Ther 18:461-468.

Pandhi P, Saha L, and Malhotra S (2001) Prolonged blockade of nitric oxide synthesis in pregnant rats as a model of pre-eclampsia. Indian J Pharmacol 33:92-95.

Parati G, Mancia G, Di Rienzo M, and Castiglioni P (2006) Point: cardiovascular variability is/is not an index of autonomic control of circulation. $J$ Appl Physiol (1985) 101:676-678, discussion 681-682.

Pavlov VA, Wang H, Czura CJ, Friedman SG, and Tracey KJ (2003) The cholinergic anti-inflammatory pathway: a missing link in neuroimmunomodulation. $\mathrm{Mol} \mathrm{Med}$ 9:125-134

Perez-Quilis C, Kingsley JD, Malkani K, Cervellin G, Lippi G, and Sanchis-Gomar F (2017) Modulation of heart rate by acute or chronic aerobic exercise. Potential effects on blood pressure control. Curr Pharm Des 23:4650-4657.

Rezk M, Gamal A, and Emara M (2015) Maternal and fetal outcome in de novo preeclampsia in comparison to superimposed preeclampsia: a two-year observational study. Hypertens Pregnancy 34:137-144.

Saha S (2005) Role of the central nucleus of the amygdala in the control of blood pressure: descending pathways to medullary cardiovascular nuclei. Clin Exp Pharmacol Physiol 32:450-456.

Saia RS, Garcia FM, and Cárnio EC (2015) Estradiol protects female rats against sepsis induced by Enterococcus faecalis improving leukocyte bactericidal activity. Steroids 102:17-26.

Sallam MY, El-Gowilly SM, Abdel-Galil AG, and El-Mas MM (2016) Central GABAA receptors are involved in inflammatory and cardiovascular consequences of endotoxemia in conscious rats. Naunyn Schmiedebergs Arch Pharmacol 389:279-288.

Sallam MY, El-Gowilly SM, El-Gowelli HM, El-Lakany MA, and El-Mas MM (2018) Additive counteraction by $\alpha 7$ and $\alpha 4 \beta 2$-nAChRs of the hypotension and cardiac sympathovagal imbalance evoked by endotoxemia in male rats. Eur $J$ Pharmacol 834:36-44.

Sallam MY, El-Gowilly SM, Fouda MA, Abd-Alhaseeb MM, and El-Mas MM (2019) Brainstem cholinergic pathways diminish cardiovascular and neuroinflammatory actions of endotoxemia in rats: role of $\mathrm{NF} \mathrm{KB} / \alpha 7 / \alpha 4 \beta 2 \mathrm{AChRs}$ signaling. Neuropharmacology 157:107683.

Sgoifo A, de Boer SF, Westenbroek C, Maes FW, Beldhuis H, Suzuki T, and Koolhaas JM (1997) Incidence of arrhythmias and heart rate variability in wild-type rats exposed to social stress. Am J Physiol 273:H1754-H1760.

Shu W, Li H, Gong H, Zhang M, Niu X, Ma Y, Zhang X, Cai W, Yang G, Wei M, et al. (2018) Evaluation of blood vessel injury, oxidative stress and circulating inflammatory factors in an L-NAME-induced preeclampsia-like rat model. Exp Ther Med 16:585-594.

Sirivelu MP, MohanKumar PS, and MohanKumar SM (2012) Differential effects of systemic interleukin-1 $\beta$ on gene expression in brainstem noradrenergic nuclei. Life Sci 90:77-81.

Smyth HS, Sleight P, and Pickering GW (1969) Reflex regulation of arterial pressure during sleep in man. A quantitative method of assessing baroreflex sensitivity. Circ Res 24:109-121.

Stein PK, Bosner MS, Kleiger RE, and Conger BM (1994) Heart rate variability: a measure of cardiac autonomic tone. Am Heart J 127:1376-1381.

Stojanovska V, Scherjon SA, and Plösch T (2016) Preeclampsia as modulator of offspring health. Biol Reprod 94:53.

Tang GJ, Kou YR, and Lin YS (1998) Peripheral neural modulation of endotoxininduced hyperventilation. Crit Care Med 26:1558-1563.

Tien Y-C, Lin J-Y, Lai C-H, Kuo C-H, Lin W-Y, Tsai C-H, Tsai F-J, Cheng Y-C, Peng W-H, and Huang C-Y (2010) Carthamus tinctorius L. prevents LPSinduced TNFalpha signaling activation and cell apoptosis through JNK1/2 NFkappaB pathway inhibition in H9c2 cardiomyoblast cells. $J$ Ethnopharmacol 130:505-513.

Vayssettes-Courchay C, Bouysset F, and Verbeuren TJ (2005) Sympathetic activation and tachycardia in lipopolysaccharide treated rats are temporally correlated and unrelated to the baroreflex. Auton Neurosci 120:35-45.

Xue P, Zheng M, Gong P, Lin C, Zhou J, Li Y, Shen L, Diao Z, Yan G, Sun H, et al. (2015) Single administration of ultra-low-dose lipopolysaccharide in rat early 
pregnancy induces TLR4 activation in the placenta contributing to preeclampsia. PLoS One 10:e124001.

Yamaguchi N, Jesmin S, Zaedi S, Shimojo N, Maeda S, Gando S, Koyama A and Miyauchi T (2006) Time-dependent expression of renal vaso-regulatory molecules in LPS-induced endotoxemia in rat. Peptides 27:2258-2270.

Zhang Y, Hua Z, Zhang K, Meng K, and Hu Y (2009) Therapeutic effects of anticoagulant agents on preeclampsia in a murine model induced by phosphatidylserine/ phosphatidylcholine microvesicles. Placenta 30:1065-1070.

Zheng L, Huang J, Su Y, Wang F, Kong H, and Xin H (2019) Vitexin ameliorates preeclampsia phenotypes by inhibiting TFPI-2 and HIF-1 $\alpha$ /VEGF in a l-NAME induced rat model. Drug Dev Res 80:1120-1127.
Zhu T, Liao X, Feng T, Wu Q, Zhang J, Cao X, and Li H (2017) Plasma monocyte chemoattractant protein 1 as a predictive marker for sepsis prognosis: a prospective cohort study. Tohoku J Exp Med 241:139-147.

Zila I, Mokra D, Kopincova J, Kolomaznik M, Javorka M, and Calkovska A (2015) Heart rate variability and inflammatory response in rats with lipopolysaccharideinduced endotoxemia. Physiol Res 64 (Suppl 5):S669-S676.

Address correspondence to: Mahmoud M. El-Mas, Department of Pharmacology and Toxicology, Faculty of Pharmacy, Alexandria University, Alazarita, Alexandria 21521, Egypt. E-mail: mahelm@hotmail.com 